\title{
LI-RADS Version 2018 Treatment Response Algorithm: Diagnostic Performance after Transarterial Radioembolization for Hepatocellular Carcinoma
}

\author{
Jongjin Yoon, Sunyoung Lee, Jaeseung Shin, Seung-seob Kim, Gyoung Min Kim, Jong Yun Won \\ All authors: Department of Radiology and Research Institute of Radiological Science, Severance Hospital, Yonsei University College of Medicine, \\ Seoul, Korea
}

Objective: To assess the diagnostic performance of the Liver Imaging Reporting and Data System (LI-RADS) version 2018 treatment response algorithm (TRA) for the evaluation of hepatocellular carcinoma (HCC) treated with transarterial radioembolization.

Materials and Methods: This retrospective study included patients who underwent transarterial radioembolization for HCC followed by hepatic surgery between January 2011 and December 2019. The resected lesions were determined to have either complete $(100 \%)$ or incomplete $(<100 \%)$ necrosis based on histopathology. Three radiologists independently reviewed the CT or MR images of pre- and post-treatment lesions and assigned categories based on the LI-RADS version 2018 and the TRA, respectively. Diagnostic performances of LI-RADS treatment response (LR-TR) viable and nonviable categories were assessed for each reader, using histopathology from hepatic surgeries as a reference standard. Inter-reader agreements were evaluated using Fleiss $\kappa$.

Results: A total of 27 patients (mean age \pm standard deviation, $55.9 \pm 9.1$ years; 24 male) with 34 lesions (15 with complete necrosis and 19 with incomplete necrosis on histopathology) were included. To predict complete necrosis, the LR-TR nonviable category had a sensitivity of $73.3-80.0 \%$ and a specificity of $78.9-89.5 \%$. For predicting incomplete necrosis, the LR-TR viable category had a sensitivity of $73.7-79.0 \%$ and a specificity of $93.3-100 \%$. Five $(14.7 \%)$ of 34 treated lesions were categorized as LR-TR equivocal by consensus, with two of the five lesions demonstrating incomplete necrosis. Interreader agreement for the LR-TR category was 0.81 (95\% confidence interval: $0.66-0.96)$.

Conclusion: The LI-RADS version 2018 TRA can be used to predict the histopathologic viability of HCCs treated with transarterial radioembolization.

Keywords: Liver neoplasms; Posttreatment imaging; Response; Radioembolization; Sensitivity and specificity

\section{INTRODUCTION}

The Liver Imaging Reporting and Data System (LI-RADS) treatment response algorithm (TRA) standardized image

Received: September 24, 2020 Revised: January 29, 2021

Accepted: February 4, 2021

Corresponding author: Sunyoung Lee, MD, PhD, Department of Radiology and Research Institute of Radiological Science, Severance Hospital, Yonsei University College of Medicine, 50-1 Yonsei-ro, Seodaemun-gu, Seoul 03722, Korea.

- E-mail: carnival0126@gmail.com

This is an 0pen Access article distributed under the terms of the Creative Commons Attribution Non-Commercial License (https://creativecommons.org/licenses/by-nc/4.0) which permits unrestricted non-commercial use, distribution, and reproduction in any medium, provided the original work is properly cited. acquisition, interpretation, reporting, and data collection in high-risk patients for hepatocellular carcinoma (HCC) who have undergone locoregional therapy (LRT) [1]. Suitable for routine clinical practice, it is designed as a simple and practical system for evaluating the treatment response of individual lesions [1]. In the LI-RADS TRA, post-treatment imaging features on contrast-enhanced CT or MRI are used to categorize treated lesions based on their LI-RADS treatment response (LR-TR), as either LR-TR nonviable (probably or definitely not viable), LR-TR equivocal (equivocally viable), or LR-TR viable (probably or definitely viable) [1]. A precise system for analyzing post-treatment imaging is crucial for clinical decision-making, as it aids in determining the presence or absence of viable tumors in 
treated lesions [2].

Transarterial radioembolization (TARE) is an increasingly utilized LRT for patients with HCC [3]. TARE, also called selective internal radiation therapy, is a transcatheter intra-arterial therapy using radioactive substances such as microspheres containing yttrium-90 (90Y), iodine-131, or similar agents $[4,5]$. The therapeutic action of TARE is predominately radiation-based, whereas its embolic effect is less prominent in minimizing alterations in hepatic arterial flow compared to other transcatheter therapies [6]. TARE is an emerging treatment option in patients who are not immediately eligible for surgery because of a large hepatic tumor extent or an insufficient residual liver volume [7]. TARE has shown promising local tumor control [8-11] and can be used on its own to achieve a complete cure or as a bridging or downstaging strategy in patients undergoing liver transplantation (LT) [3].

Several studies have evaluated the performance of LIRADS TRA for the assessment of HCCs treated with various types of LRTs, including radiofrequency ablation, microwave ablation, transcatheter bland arterial embolization, and chemoembolization with or without drug-eluting beads [1216]. However, to the best of our knowledge, the diagnostic performance of LI-RADS TRA for the evaluation of HCCS treated with radiation-based LRTs such as TARE has not yet been reported. Therefore, the purpose of our study was to assess the diagnostic performance of LI-RADS version 2018 TRA for the evaluation of HCCs treated with TARE.

\section{MATERIALS AND METHODS}

This retrospective study was approved by the Institutional Review Board of Severance Hospital, and the requirement to obtain informed consent was waived (IRB No. 4-20200279). The study protocol conformed to the ethical guidelines of the 1975 Declaration of Helsinki.

\section{Patients}

From a search of our institution's databases, we identified 53 patients who first underwent TARE and then subsequent hepatic surgery (resection or LT) between January 2011 and December 2019. The inclusion criteria were as follows: 1) age 18 years or older; 2) patients at high risk for HCC with liver cirrhosis or chronic hepatitis B viral infection; and 3) patients who underwent multiphase CT or MRI before and after TARE. The exclusion criteria were as follows: 1) patients who underwent combined treatment with other LRTs or systemic treatment $(n=11) ; 2)$ no preoperative multiphase CT or MRI examination performed within 1 month before surgery $(n=9)$; and 3) patients who were diagnosed with cholangiocarcinoma, combined hepatocellular-cholangiocarcinoma, or metastasis on histopathology after surgery $(n=3)$ (Fig. 1).

\section{Lesion Registry and Data Collection}

A board-certified abdominal radiologist (with 10 years' experience) marked individual lesions to be reviewed on pre- and post-treatment CT or MR imaging, reported the

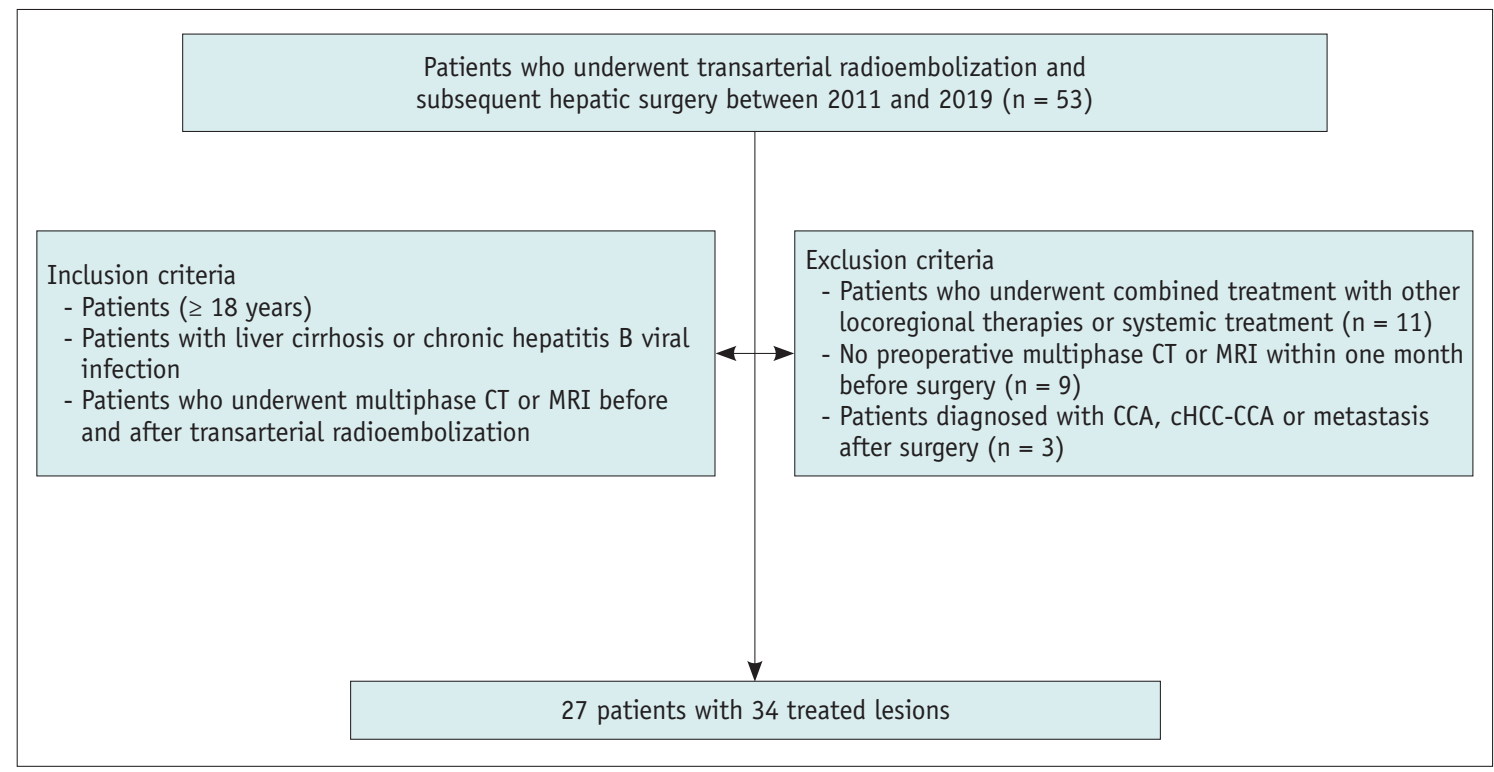

Fig. 1. Flowchart of the study population. $\mathrm{CCA}=$ cholangiocarcinoma, $\mathrm{CHCC}-\mathrm{CCA}=$ combined hepatocellular-cholangiocarcinoma 
lesions based on segmental location, and provided a list for review. MR images were preferentially considered for image analysis. CT images were used when there were no available MR images. Clinical and laboratory data were collected from electronic medical records of each patient, including age at TARE, sex, etiology of liver disease, serum total bilirubin, alpha-fetoprotein, and protein induced by vitamin $\mathrm{K}$ absence or antagonist-II.

Histopathological data were extracted from pathologic reports. Collected histopathological data included segmental tumor location, histopathologic diagnosis, and histopathologic tumor necrosis (incomplete [ $<100 \%$ ] necrosis vs. complete [100\%] necrosis).

\section{CT and MRI Examinations}

Multiphase CT was performed using 64- or 128-channel multidetector CT scanners (Sensation 64 or SOMATOM Definition Flash, Siemens Healthineers; LightSpeed VCT, GE Healthcare). The protocol included precontrast, late arterial, portal venous, and delayed phases. After the precontrast scan, contrast-enhanced CT was performed after intravenous administration of $2.0 \mathrm{~mL} / \mathrm{kg}$ iodinated contrast media for a fixed injection duration of 30 seconds, followed by a 20 $\mathrm{mL}$ saline flush. The late arterial phase scan was obtained 18 seconds after the attenuation value reached 100 Hounsfield unit at the abdominal aorta, using the bolustracking technique. The portal venous phase images were acquired 30 seconds after obtaining the late arterial phase, and the delayed phase images were acquired 150 seconds after obtaining the portal phase images. The CT acquisition parameters are presented in Supplementary Table 1.

MRI was performed using a 3T scanner (Magnetom Trio Tim, Siemens Healthineers; Intera Achieva, Ingenia, or Ingenia CX, Philips Healthcare). The protocol included acquisition of dual-echo spoiled gradient-echo T1-weighted in-phase and opposed-phase images, single-shot and multishot turbo spin-echo T2-weighted images, and diffusionweighted imaging with single-shot echo-planar images at b-values of $0,50,400$, and $800 \mathrm{~s} / \mathrm{mm}^{2}$. Dynamic fatsuppressed spoiled gradient-echo T1-weighted images were obtained before and after administering an extracellular contrast agent (ECA) (gadoterate meglumine, Dotarem, Guerbet SA) or a hepatobiliary agent (HBA) (gadoxetate disodium, Primovist, Bayer Pharma AG). Bolus injections of $0.2 \mathrm{~mL} / \mathrm{kg}$ of gadoterate meglumine at a rate of $2.0 \mathrm{~mL} / \mathrm{s}$ or of $0.1 \mathrm{~mL} / \mathrm{kg}$ gadoxetate disodium at a rate of $1.0 \mathrm{~mL} / \mathrm{s}$ were performed, followed by a 20 -
$\mathrm{mL}$ saline flush. The choice of MRI contrast agents was made at the discretion of the physicians. Arterial phase scanning was initiated using the test-bolus or bolustracking techniques, and the portal venous phase (60 seconds), 3-minute delayed phase (transitional phase on HBA-MRI), and 20-minute hepatobiliary phase images (only after HBA administration) were evaluated. Detailed MRI parameters are presented in Supplementary Table 2.

\section{TARE with Yttrium-90}

All patients underwent pretreatment angiography for assessing the suitability of treatment and for determining dose calculations. Celiac, common hepatic, and conebeam $\mathrm{CT}$ arteriography were performed to identify feeding arteries supplying the tumor and to determine the target artery of radioembolization. Technetium- $99 \mathrm{~m}$-labeled macroaggregated albumin was scanned to quantify the lung shunt fraction. TARE was performed with resin microspheres (SIR-Spheres, Sirtex Medical) or glass microspheres (TheraSphere, Biocompatibles UK Ltd) loaded with ${ }^{90} \mathrm{Y}$. The target-absorbed radiation dose of the normal liver and lung was determined to not exceed $70 \mathrm{~Gy}$ and $25 \mathrm{~Gy}$, respectively. TARE was performed only once in each patient.

\section{Image Analysis}

Two board-certified abdominal radiologists (reader 1 and reader 2, with nine and five years' experience, respectively) and a radiology resident (reader 3, 2nd-year resident) independently reviewed each patient's pre- and posttreatment CT or MR imaging and evaluated all lesions using the CT/MRI LI-RADS version 2018 for pretreatment images and the TRA for post-treatment images. All readers were blinded to the patients' clinical information and radiologic and histopathologic reports, but were informed that all patients had undergone TARE for HCC.

On pretreatment images, all readers assessed lesion size and major imaging features, including nonrim arterial phase hyperenhancement (APHE), non-peripheral washout, and enhancing capsule appearance. LI-RADS category M (LR$M$; probably or definitely malignant but not HCC specific) criteria including the targetoid mass and tumor in vein were also evaluated. A pretreatment LI-RADS category was assigned to each lesion based on the CT/MRI LI-RADS version 2018 [1].

On post-treatment/preoperative images, all readers assessed the presence or absence of treatment response features and measured whole lesion size and viable or 
equivocally viable tumor size. The LI-RADS treatment response category was determined based on the following treatment response features: 1 ) LR-TR nonviable (probably or definitely not viable): no lesional enhancement or a treatment-specific expected enhancement pattern including a thin rim of enhancement around the treated tumor, geographic zone of perilesional enhancement without washout appearance, or non-mass-like foci of perilesional enhancement without washout appearance; 2) LR-TR equivocally viable: indeterminate enhancement defined as an enhancement atypical for treatment-specific expected enhancement pattern and not meeting criteria for being probably or definitely viable; or 3) LR-TR visible (probably or definitely viable): nodular, mass-like, or irregular thick tissue (NMLIT) in or along the treated lesion with any of the following, APHE, washout appearance, or enhancement similar to pretreatment [1].

APHE was defined as enhancement unequivocally higher attenuation or intensity than the liver in the arterial phase [1]. When a lesion showed high attenuation or signal intensity on precontrast T1-weighted image, APHE was evaluated by subtracting the arterial phase image [1]. Washout appearance was determined on portal venous or delayed phases on CT or MRI examinations with ECA, but only on portal venous phase in MRI examinations with HBA [1]. In cases with discrepancies between the three readers, consensus was determined by agreement between at least two readers.

\section{Statistical Analysis}

Descriptive data are presented as numbers and percentages. Continuous variables are reported as means \pm standard deviations or medians and interquartile ranges (IQR). The sensitivity, specificity, accuracy, positive predictive value (PPV), and negative predictive value (NPV) of LR-TR categories for predicting histopathologic complete or incomplete necrosis were calculated for each reader. Inter-reader agreements for pre-and post-treatment imaging features and for assigned categories were evaluated using the Fleiss $\kappa$, with $95 \%$ confidence intervals (CIs). The $\kappa$ value was interpreted as slight (0.01-0.20), fair (0.21$0.40)$, moderate $(0.41-0.60)$, substantial $(0.61-0.80)$, or almost perfect (0.81-1.00) [17]. Statistical analyses were performed using SPSS version 26.0 (IBM Corp.) and MedCalc version 16.2.1 (MedCalc Software).

\section{RESULTS}

\section{Characteristics of Patients and Lesions}

A total of 27 patients (mean age, 55.9 years; 24 men and three women) with 34 lesions were included in this study. The characteristics of the patients and lesions are summarized in Table 1. Hepatitis B viral infection (92.6\%, 25 of 27) was the predominant etiology of liver disease in these patients. Twenty-one (77.8\%) patients had one lesion, and six patients $(22.2 \%)$ had two or three lesions. Twenty patients $(74.1 \%)$ underwent hepatic resections and seven (25.9\%) underwent LT. The median interval between TARE and hepatic surgery was 240 days (IQR, 160-486 days).

The pretreatment imaging examinations included two

Table 1. Characteristics of Patients and Lesions prior to Transarterial Radioembolization

\begin{tabular}{|c|c|}
\hline Characteristics & Value \\
\hline Patients & $\mathrm{n}=27$ \\
\hline Age at time of TARE, year* & $55.9 \pm 9.1$ \\
\hline Sex, male:female & $24: 3$ \\
\hline \multicolumn{2}{|l|}{ Etiology of liver disease } \\
\hline Hepatitis B & $25(92.6)$ \\
\hline Alcohol & $2(7.4)$ \\
\hline Total bilirubin level, $\mathrm{mg} / \mathrm{dL}^{\dagger}$ & $0.6(0.4-0.9)$ \\
\hline $\mathrm{AFP}, \mathrm{ng} / \mathrm{mL}^{\dagger}$ & $14.0(3.6-32.7)$ \\
\hline PIVKA-II, mAU/mL ${ }^{\dagger}$ & $2663(180-7752)$ \\
\hline \multicolumn{2}{|l|}{ Operation type } \\
\hline Resection & $20(74.1)$ \\
\hline Transplantation & $7(25.9)$ \\
\hline Lesions & $\mathrm{n}=34$ \\
\hline \multicolumn{2}{|c|}{ Pretreatment LI-RADS category by consensus } \\
\hline LR-4 & $3(8.8)$ \\
\hline LR-5 & $26(76.5)$ \\
\hline LR-M & $2(5.9)$ \\
\hline LR-TIV & $3(8.8)$ \\
\hline \multicolumn{2}{|c|}{ Pretreatment lesion characteristics by consensus } \\
\hline Size, $\mathrm{mm}^{*}$ & $70.1 \pm 30.4$ \\
\hline Nonrim APHE & $32(94.1)$ \\
\hline Nonperipheral washout appearance & $29(85.3)$ \\
\hline Enhancing capsule appearance & $22(64.7)$ \\
\hline Targetoid mass & $2(5.9)$ \\
\hline TIV & $3(8.8)$ \\
\hline
\end{tabular}

Unless otherwise indicated, data are numbers of patients and lesions, with percentages in parentheses. * Data are mean \pm standard deviation, ${ }^{\dagger}$ Data are median with interquartile range in parentheses. AFP = alpha-fetoprotein, APHE = arterial phase hyperenhancement, LI-RADS = Liver Imaging Reporting and Data System, LR = Liver Imaging Reporting and Data System, TIV = tumor in vein 
CT examinations, four MRI examinations with ECA, and 21 MRI examinations with HBA. The pretreatment LI-RADS categories for the 34 lesions were determined by consensus to be as follows: the majority of lesions ( 26 of $34,76.5 \%$ ) were classified as LR-5, three lesions (8.8\%) as LR-4, two lesions (5.9\%) as LR-M, and three lesions (8.8\%) as LR-TIV. The mean size of pretreatment lesions was $70.1 \mathrm{~mm}$ (range, 10-123 mm).

\section{Post-Treatment Imaging and Histopathologic Tumor Necrosis}

The post-treatment imaging examinations included $10 \mathrm{CT}$ examinations, one MRI examination with ECA, and 16 MRI examinations with HBA. The post-treatment LR-TR categories for lesion characteristics by consensus are summarized in Table 2. In this study, 44.1\% (15 of 34) of treated lesions were categorized as LR-TR nonviable by consensus, with 12 of the 15 demonstrating complete necrosis on histopathology. There were $14.7 \%$ (5 of 34 ) of treated lesions categorized as LR-TR equivocal by consensus, with two of the five lesions demonstrating incomplete necrosis on histopathology. Finally, $41.2 \%$ (14 of 34) of treated lesions were categorized as LR-TR viable by consensus, with all these lesions confirmed to have incomplete necrosis on histopathology. All 14 lesions categorized as LR-TR viable showed NMLIT with APHE. NMLIT with washout appearance and NMLIT with enhancement similar to pretreatment were detected in $57.1 \%(8 / 14)$ and $57.1 \%(8 / 14)$ of treated lesions, respectively.

\section{Diagnostic Performances of LR-TR Categories}

The diagnostic performances of LR-TR categories for predicting complete and incomplete necrosis on histopathology are presented in Table 3 and Supplementary Table 3. For predicting complete necrosis on histopathology, the LR-TR nonviable category had a sensitivity ranging from $73.3 \%$ to $80.0 \%$ (12 of 15,11 of 15 , and 11 of 15 for readers 1,2 , and 3 , respectively) and a specificity ranging from $78.9 \%$ to $89.5 \%$ (15 of 19,17 of 19 , and 16 of 19 for readers 1,2 , and 3, respectively), with a PPV ranging from $75.0 \%$ to $84.6 \%$ (12 of 16,11 of 13 , and 11 of 14 for readers 1,2 , and 3 , respectively) and an NPV ranging from $80.0 \%$ to $83.3 \%$ ( 15 of 18,17 of 21 , and 16 of 20 for readers 1, 2, and 3, respectively) (Fig. 2). To predict incomplete necrosis, the LR-TR viable category had a sensitivity ranging from $73.7 \%$ to $79.0 \%$ (14 of 19,15 of 19 , and 14 of 19 for readers 1,2 , and 3, respectively) and a specificity ranging from $93.3 \%$ to $100 \%$ (15 of 15,14 of 15 , and 14 of 15 for readers 1,2 , and 3, respectively), with a PPV ranging from $93.3 \%$ to $100 \%$ (14 of 14,15 of 16 , and 14 of 15 for readers 1,2 , and 3, respectively) and an NPV ranging from $73.7 \%$ to $77.8 \%$ (15 of 20,14 of 18 , and 14 of 19 for readers 1, 2, and 3, respectively) (Fig. 3).

\section{Table 2. Post-Treatment Lesion Characteristics by Consensus LR-TR Category}

\begin{tabular}{|c|c|c|c|}
\hline Characteristic & $\begin{array}{l}\text { LR-TR Nonviable } \\
\qquad(\mathrm{n}=15)\end{array}$ & $\begin{array}{l}\text { LR-TR Equivocal } \\
\qquad(n=5)\end{array}$ & $\begin{array}{l}\text { LR-TR Viable } \\
\quad(n=14)\end{array}$ \\
\hline \multicolumn{4}{|l|}{ Histopathologic tumor necrosis } \\
\hline Complete $(100 \%)$ necrosis & $80.0(12 / 15)$ & $60.0(3 / 5)$ & 0 \\
\hline Incomplete $(<100 \%)$ necrosis & $20.0(3 / 15)$ & $40.0(2 / 5)$ & $100(14 / 14)$ \\
\hline \multicolumn{4}{|l|}{ Post-treatment imaging characteristics } \\
\hline \multicolumn{4}{|l|}{ Treatment response features } \\
\hline No lesional enhancement & $13.3(2 / 15)$ & 0 & 0 \\
\hline Treatment-specific expected enhancement pattern & $86.7(13 / 15)$ & 0 & 0 \\
\hline NMLIT with APHE & 0 & 0 & $100(14 / 14)$ \\
\hline NMLIT with washout appearance & 0 & 0 & $57.1(8 / 14)$ \\
\hline NMLIT with enhancement similar to pretreatment & 0 & 0 & $57.1(8 / 14)$ \\
\hline Indeterminate enhancement* & 0 & $100(5 / 5)$ & 0 \\
\hline \multicolumn{4}{|l|}{ Size } \\
\hline Whole lesion longest measurement, $\mathrm{mm}^{\dagger}$ & $37.0 \pm 20.0$ & $55.6 \pm 15.3$ & $59.1 \pm 24.3$ \\
\hline Viable or equivocally viable tumor size, $\mathrm{mm}^{\dagger}$ & $0 \pm 0$ & $8.2 \pm 1.7$ & $37.9 \pm 22.3$ \\
\hline
\end{tabular}

Unless otherwise specified, data are percentages and data in parentheses are raw data. * Indeterminate enhancement defined as an enhancement atypical for treatment-specific expected enhancement pattern and not meeting criteria being for probably or definitely viable, ${ }^{\dagger}$ Data are mean \pm standard deviation. APHE $=$ arterial phase hyperenhancement, LR-TR = Liver Imaging Reporting and Data System treatment response, NMLIT = nodular, masslike, or irregular thick tissue in or along the treated lesion 
Table 3. Diagnostic Performances for Predicting Histopathologic Incomplete Tumor Necrosis in the LR-TR Viable Category and Complete Necrosis in the LR-TR Nonviable Category

\begin{tabular}{|c|c|c|c|c|c|}
\hline & Sensitivity (\%) & Specificity (\%) & Accuracy $(\%)$ & PPV $(\%)$ & NPV $(\%)$ \\
\hline \multicolumn{6}{|c|}{ LR-TR nonviable category as a predictor of complete $(100 \%)$ necrosis } \\
\hline \multirow{2}{*}{ Reader 1} & $80.0(12 / 15)$ & $78.9(15 / 19)$ & $79.4(27 / 34)$ & $75.0(12 / 16)$ & $83.3(15 / 18)$ \\
\hline & {$[51.9,95.7]$} & {$[54.4,93.9]$} & {$[62.1,91.3]$} & {$[47.6,92.7]$} & {$[58.6,96.4]$} \\
\hline \multirow{2}{*}{ Reader 2} & $73.3(11 / 15)$ & $89.5(17 / 19)$ & $82.4(28 / 34)$ & $84.6(11 / 13)$ & $81.0(17 / 21)$ \\
\hline & {$[44.9,92.2]$} & {$[66.9,98.7]$} & {$[65.5,93.2]$} & {$[54.6,98.1]$} & {$[58.1,94.6]$} \\
\hline \multirow{2}{*}{ Reader 3} & $73.3(11 / 15)$ & $84.2(16 / 19)$ & $79.4(27 / 34)$ & $78.6(11 / 14)$ & $80.0(16 / 20)$ \\
\hline & {$[44.9,92.2]$} & {$[60.4,96.6]$} & {$[62.1,91.3]$} & {$[49.2,95.3]$} & {$[56.3,94.3]$} \\
\hline \multicolumn{6}{|c|}{ LR-TR viable category as a predictor of incomplete $(<100 \%)$ necrosis } \\
\hline \multirow{2}{*}{ Reader 1} & $73.7(14 / 19)$ & $100(15 / 15)$ & $85.3(29 / 34)$ & $100(14 / 14)$ & $75.0(15 / 20)$ \\
\hline & {$[48.8,90.9]$} & {$[78.2,100]$} & {$[68.9,95.1]$} & {$[76.8,100]$} & {$[50.9,91.3]$} \\
\hline \multirow{2}{*}{ Reader 2} & $79.0(15 / 19)$ & $93.3(14 / 15)$ & $85.3(29 / 34)$ & $93.8(15 / 16)$ & $77.8(14 / 18)$ \\
\hline & {$[54.4,94.0]$} & {$[68.1,99.8]$} & {$[68.9,95.1]$} & {$[69.8,99.8]$} & {$[52.4,93.6]$} \\
\hline \multirow{2}{*}{ Reader 3} & $73.7(14 / 19)$ & $93.3(14 / 15)$ & $82.4(28 / 34)$ & $93.3(14 / 15)$ & $73.7(14 / 19)$ \\
\hline & {$[48.8,90.9]$} & {$[68.1,99.8]$} & {$[65.5,93.2]$} & {$[68.1,99.8]$} & {$[48.8,90.9]$} \\
\hline
\end{tabular}

Data in parenthesis are the raw data, and data in brackets are the $95 \%$ confidence interval. LR-TR $=$ Liver Imaging Reporting and Data System treatment response, NPV $=$ negative predictive value, $P P V=$ positive predictive value

\section{Inter-Reader Agreement}

Inter-reader agreement for pre- and post-treatment imaging features are summarized in Supplementary Table 4. Inter-reader agreement for post-treatment LR-TR category was almost perfect ( $\kappa=0.81 ; 95 \%$ CI: $0.66,0.96)$. For post-treatment imaging features, NMLIT with APHE showed the highest agreement ( $\kappa=0.92 ; 95 \%$ CI: 0.73 .1 .12$)$, followed by NMLIT with enhancement similar to pretreatment $(\kappa=0.75 ; 95 \%$ CI: 0.55, 0.94). Readers were in the lowest agreement regarding no lesional enhancement $(\kappa=0.22$; 95\% CI: $0.03,0.41)$.

\section{DISCUSSION}

In this study of patients with HCC treated with TARE, the LI-RADS version 2018 TRA performed well for predicting histopathologic complete necrosis (LR-TR nonviable category: sensitivity $=73.3-80.0 \%$; specificity $=78.9-$ $89.5 \%$ ) and incomplete necrosis (LR-TR viable category: sensitivity $=73.7-79.0 \%$; specificity $=93.3-100 \%$ ). Five $(14.7 \%)$ of 34 treated lesions were assigned a LR-TR equivocal category by consensus, and two of these lesions showed incomplete necrosis on histopathology.

Previous studies have assessed the performance of the LR-TR nonviable category for other types of LRTs. In a study of bland arterial embolization, Shropshire et al. [12] showed that less than half of the lesions (38-46\%) classified as LR-TR nonviable had complete histopathologic tumor necrosis. Seo et al. [14] reported that approximately two-thirds of LR-TR nonviable lesions (60-64\%) were confirmed to have complete necrosis after transcatheter chemoembolization or radiofrequency ablation. In our study, $80.0 \%$ (12 of 15) of TARE-treated lesions categorized as LR-TR nonviable by consensus were completely necrotic on histopathology, which is a higher percentage than previously reported in studies investigating other types of LRTs $[13,14]$. This difference may be related to the characteristics of the individual treatment modality. In addition, the NPV of the LR-TR nonviable category for prediction of complete tumor necrosis ranged from $80.0 \%$ to $83.3 \%$. This high NPV suggests that it may be prudent to consider continued imaging surveillance without additional treatments in patients with this treatment response category after TARE [2].

The LR-TR viable category has also been evaluated in previous studies of LRTs. While we found that the LR-TR viable category had a sensitivity of $73.7 \%$ to $79.0 \%$ for predicting incomplete tumor necrosis after TARE, previously reported studies utilizing conventional chemoembolization and post-treatment CT imaging evaluations showed lower sensitivities for this category $[14,16]$. It has been suggested that beam-hardening artifacts of lipiodolized nodules after conventional chemoembolization may obscure precise evaluation of APHE on CT imaging and may underestimate residual viable tumors [18]. Our study also demonstrated that the LR-TR viable category had a high specificity of $93.3 \%$ to $100 \%$. This finding is comparable to the specificity values reported in previous studies 
evaluating LI-RADS TRA in patients with HCC and comparing tumor viability to histopathology [13-15]. The LI-RADS TRA may achieve this high specificity by preventing falsepositive diagnoses based on treatment-specific changes
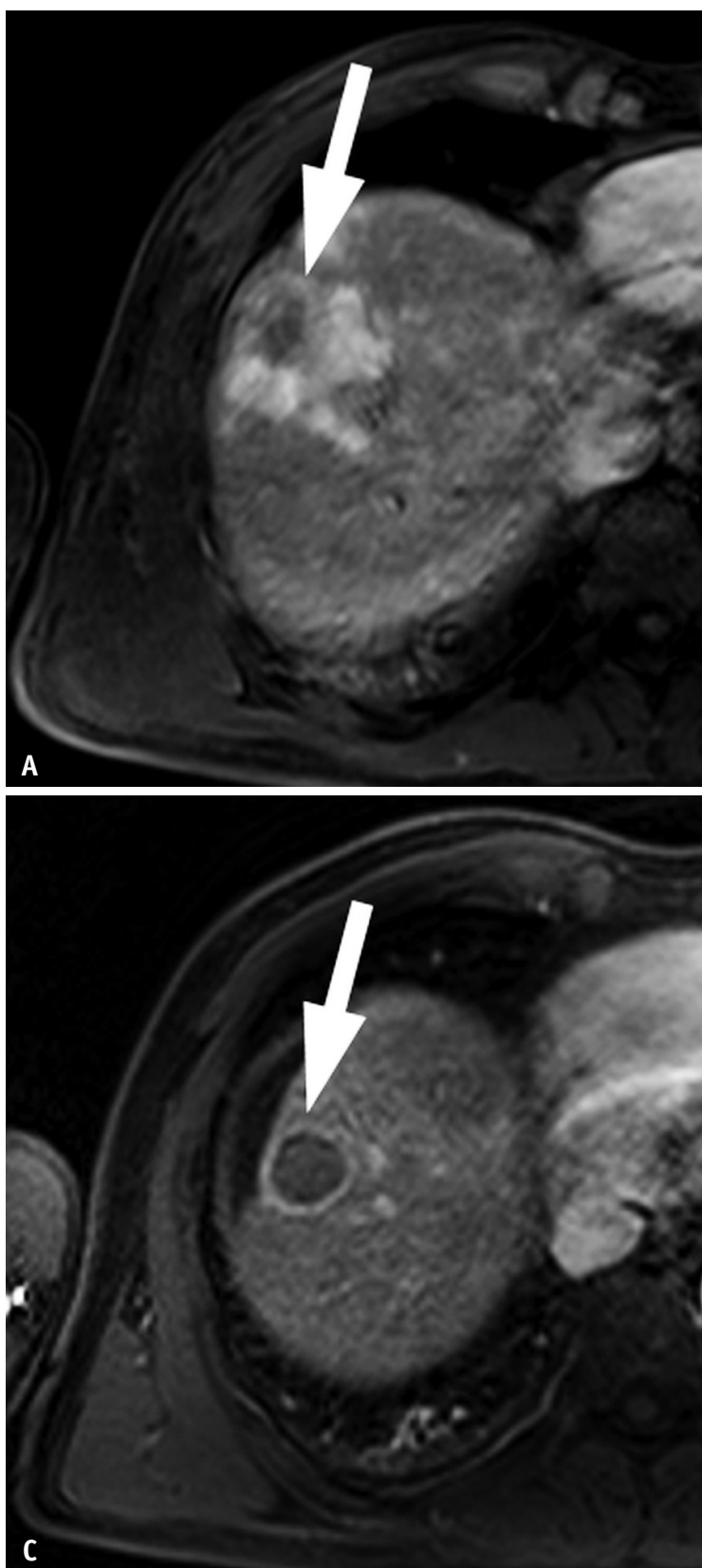

[16]. In clinical practice, the high specificity of this algorithm for identifying viable tumors in treated lesions is crucial for avoiding unnecessary additional treatments.

Among the characteristic imaging findings for the LR-
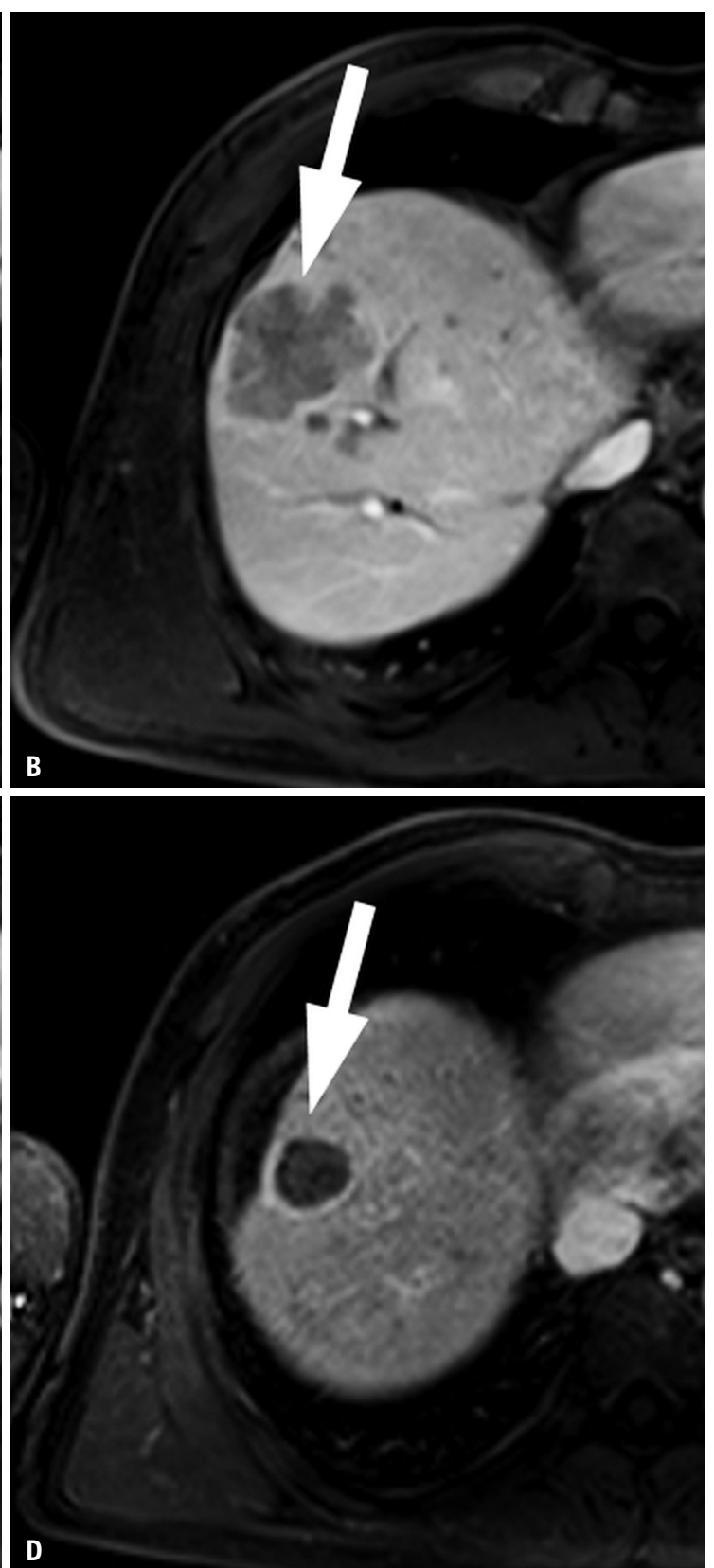

Fig. 2. Axial gadoxetate disodium-enhanced magnetic resonance images of a 53-year-old male with chronic hepatitis B and hepatocellular carcinoma.

A. Pretreatment arterial phase T1-weighted 3D gradient-echo image. B. Pretreatment portal venous phase T1-weighted 3D gradient-echo image. C. Post-treatment arterial phase T1-weighted 3D gradient-echo image. D. Post-treatment portal venous phase T1-weighted 3D gradient-echo image. A, B. Pretreatment (A) arterial and (B) portal venous phase T1-weighted 3D gradient-echo images show a 57-mm lesion with arterial phase hyperenhancement, non-peripheral washout, and enhancing capsule appearance (arrows), categorized as LR- 5 by the LI-RADS version 2018. C, D. Post-treatment (C) arterial and (D) portal venous phase T1-weighted 3D gradient-echo images, obtained 186 days after transarterial radioembolization, show a decrease in the size of the treated lesion to $23 \mathrm{~mm}$, with no residual enhancement except a thin enhancing rim, without any lesion-associated washout (arrows). This lesion is categorized as LI-RADS treatment response nonviable by the LI-RADS version 2018 treatment response algorithm and was confirmed to have complete necrosis on histopathology after hepatic resection. LI-RADS $=$ Liver Imaging Reporting and Data System, LR = Liver Imaging Reporting and Data System, 3D = three-dimensional 
TR viable category, our study found that NMLIT with APHE was the most frequently observed feature in patients with HCC treated with TARE, a finding that is consistent with a previous study evaluating imaging findings after locoablative therapy [13]. Although the LI-RADS TRA assesses tumor viability using several imaging features,
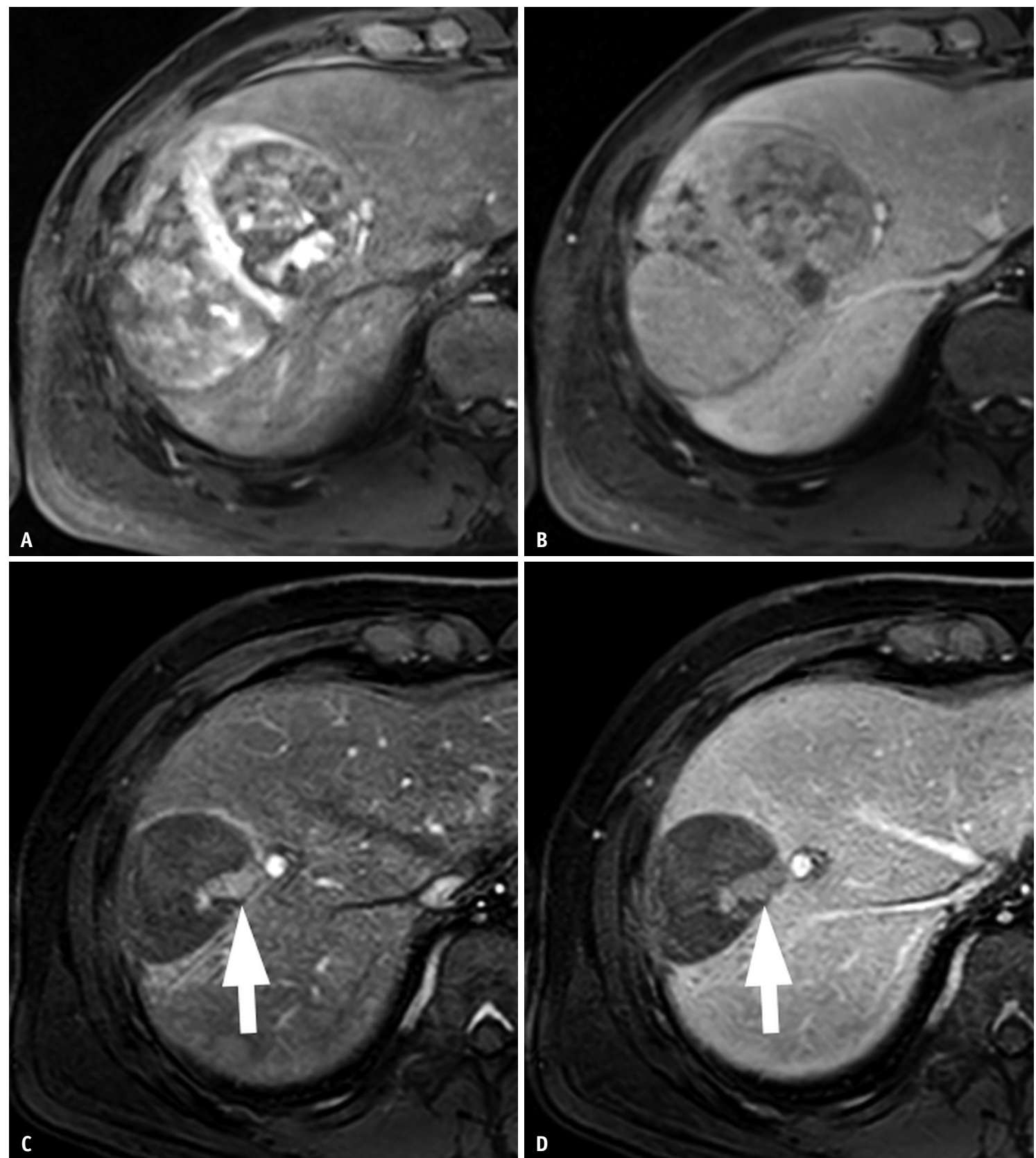

Fig. 3. Axial gadoxetate disodium-enhanced magnetic resonance images of a 48-year-old male with chronic hepatitis B and hepatocellular carcinoma.

A. Pretreatment arterial phase T1-weighted 3D gradient-echo image. B. Pretreatment portal venous phase T1-weighted 3D gradient-echo image. C. Post-treatment arterial phase T1-weighted 3D gradient-echo image. D. Post-treatment portal venous phase T1-weighted 3D gradient-echo image. A, B. Pretreatment (A) arterial and (B) portal venous phase T1-weighted 3D gradient-echo images show a 99-mm lesion with APHE and nonperipheral washout appearance, categorized as LR-5 by LI-RADS version 2018. C, D. Post-treatment (C) arterial and (D) portal venous phase T1weighted 3D gradient-echo images, obtained 378 days after transarterial radioembolization, show a decrease in the size of the treated lesion to 51 $\mathrm{mm}$, with a 22-mm nodular, mass-like, or irregular thick tissue in the treated lesion demonstrating APHE and a washout appearance (arrows). This lesion is categorized as LI-RADS treatment response viable by the LI-RADS version 2018 treatment response algorithm and was confirmed to have incomplete necrosis on histopathology after hepatic resection. APHE $=$ arterial phase hyperenhancement, LI-RADS $=$ Liver Imaging Reporting and Data System, LR = Liver Imaging Reporting and Data System, 3D = three-dimensional 
including NMLIT with APHE, washout appearance, and enhancement similar to pretreatment, NMLIT with APHE appears to be predominantly used for identifying incomplete tumor necrosis, with other imaging features providing complementary information.

The LR-TR equivocal category reflects uncertainty in assessing tumor viability on post-treatment imaging, with lesions showing enhancement that is atypical for treatment-specific expected enhancement patterns and does not meet the criteria for being probably or definitely viable. Our study demonstrated that $40 \%$ (two of five) of LR-TR equivocal lesions were identified to have incomplete necrosis after TARE, and 60\% (three of five) were confirmed to have complete necrosis on histopathology. After TARE, patchy arterial enhancement can be seen in treated tumors due to post-treatment inflammatory changes [19]. Evolving parenchymal abnormalities surrounding the treated tumors may also confound the apparent attenuation/signal and enhancement characteristics of lesions [20]. These postTARE changes may cause lesions to be classified as LR-TR equivocal, though they do not have residual viable tumor identified on histopathology.

This study had several limitations. The retrospective, single-center design introduced an unavoidable selection bias. In addition, the number of patients and lesions included in this study was relatively small. In addition, our study was restricted to patients with $\mathrm{HCC}$ who were treated with TARE and who underwent subsequent hepatic surgery; accordingly, histopathologic necrosis could be used as a reference standard. Our exclusive focus on this patient population can be considered a strength of this study, as it provided potentially valuable information about the performance of the LI-RADS TRA for this specific type of LRT, which has not previously been evaluated. Alternatively, it could be considered a weakness, as we did not evaluate the performance of the LI-RADS TRA for other types of LRTs, including locoablative therapies, other transcatheter therapies, and external beam radiation therapy. Another limitation of our study was the use of heterogeneous imaging modalities (i.e., CT, MRI with ECA, and MRI with $\mathrm{HBA}$ ) used for image analysis. It is already known that background liver affects the detection of LIRADS imaging features in CT rather than in MRI [21]. In addition, subtraction AP imaging of MRI with HBA improves diagnostic performance of HCC in the LR-5 category [22], whereas subtraction AP imaging is not available on CT. Thus, the heterogeneous imaging modality itself provides heterogeneous diagnostic performance. Furthermore, we were not able to explore differences in the performance of the LI-RADS TRA based on imaging modality (i.e., CT vs. MRI) due to insufficient numbers. Further prospective studies conducted at multiple centers with larger numbers of patients and lesions are needed to determine and compare the performance of LI-RADS TRA for different types of LRTs and imaging modalities. Lastly, the heterogeneous readers' experience of image interpretation in our study was another limitation.

In conclusion, our study demonstrates that the LI-RADS version 2018 TRA shows good performance for HCCs treated with TARE when lesions are assessed as LR-TR viable or nonviable. The LI-RADS version 2018 TRA can be used to predict the histopathologic viability of HCCs treated with TARE. Further prospective studies with a large multicenter multi-reader setting are required to validate the performance of the LI-RADS version 2018 TRA in patients with HCC treated with TARE and other LRTs. A comparative study between imaging modalities and techniques will also be valuable.

\section{Supplement}

The Supplement is available with this article at https://doi.org/10.3348/kjr.2020.1159.

\section{Conflicts of Interest}

The authors have no potential conflicts of interest to disclose.

\section{Author Contributions}

Conceptualization: Jongjin Yoon, Sunyoung Lee. Data curation: Jongjin Yoon, Sunyoung Lee, Jaeseung Shin. Formal analysis: Jongjin Yoon, Sunyoung Lee, Jaeseung Shin. Investigation: Seung-seob Kim. Methodology: Jongjin Yoon, Sunyoung Lee. Project administration: Sunyoung Lee. Supervision: Sunyoung Lee. Validation: Seung-seob Kim, Gyoung Min Kim, Jong Yun Won. Visualization: Seung-seob Kim, Gyoung Min Kim, Jong Yun Won. Writing-original draft: Jongjin Yoon, Sunyoung Lee. Writing-review \& editing: Jongjin Yoon, Sunyoung Lee.

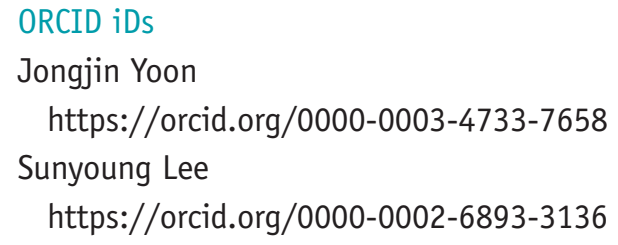


Jaeseung Shin

https://orcid.org/0000-0002-6755-4732

Seung-seob Kim

https://orcid.org/0000-0001-6071-306X

Gyoung Min Kim

https://orcid.org/0000-0001-6768-4396

Jong Yun Won

https://orcid.org/0000-0002-8237-5628

\section{REFERENCES}

1. American College of Radiology. CT/MRI Liver Imaging Reporting and Data System version 2018. Acr.org Web site. https://www.acr.org/Clinical-Resources/Reporting-and-DataSystems/LI-RADS/CT-MRI-LI-RADS-v2018. Accessed August 12,2018

2. Gervais DA. LI-RADS treatment response algorithm: performance and diagnostic accuracy. Radiology 2019;292:235-236

3. Marrero JA, Kulik LM, Sirlin CB, Zhu AX, Finn RS, Abecassis $M M$, et al. Diagnosis, staging, and management of hepatocellular carcinoma: 2018 practice guidance by the American Association for the Study of Liver Diseases. Hepatology 2018;68:723-750

4. European Association for the Study of the Liver. EASL clinical practice guidelines: management of hepatocellular carcinoma. J Hepatol 2018;69:182-236

5. Kim HC. Radioembolization for the treatment of hepatocellular carcinoma. Clin Mol Hepatol 2017;23:109-114

6. Sangro B, Iñarrairaegui M, Bilbao JI. Radioembolization for hepatocellular carcinoma. J Hepatol 2012;56:464-473

7. Sacco R, Mismas V, Marceglia S, Romano A, Giacomelli L, Bertini M, et al. Transarterial radioembolization for hepatocellular carcinoma: an update and perspectives. World J Gastroenterol 2015;21:6518-6525

8. Kulik LM, Carr BI, Mulcahy MF, Lewandowski RJ, Atassi B, Ryu RK, et al. Safety and efficacy of 90 Y radiotherapy for hepatocellular carcinoma with and without portal vein thrombosis. Hepatology 2008;47:71-81

9. Hilgard P, Hamami M, Fouly AE, Scherag A, Müller S, Ertle J, et al. Radioembolization with yttrium-90 glass microspheres in hepatocellular carcinoma: European experience on safety and long-term survival. Hepatology 2010;52:1741-1749

10. Salem R, Lewandowski RJ, Mulcahy MF, Riaz A, Ryu RK, Ibrahim $S$, et al. Radioembolization for hepatocellular carcinoma using Yttrium-90 microspheres: a comprehensive report of long-term outcomes. Gastroenterology 2010;138:52-64

11. Sangro B, Carpanese L, Cianni R, Golfieri R, Gasparini D,
Ezziddin S, et al. Survival after yttrium-90 resin microsphere radioembolization of hepatocellular carcinoma across Barcelona clinic liver cancer stages: a European evaluation. Hepatology 2011;54:868-878

12. Shropshire EL, Chaudhry M, Miller CM, Allen BC, Bozdogan E, Cardona DM, et al. LI-RADS treatment response algorithm: performance and diagnostic accuracy. Radiology 2019;292:226-234

13. Chaudhry M, McGinty KA, Mervak B, Lerebours R, Li C, Shropshire E, et al. The LI-RADS version 2018 MRI treatment response algorithm: evaluation of ablated hepatocellular carcinoma. Radiology 2020;294:320-326

14. Seo N, Kim MS, Park MS, Choi JY, Do RKG, Han K, et al. Evaluation of treatment response in hepatocellular carcinoma in the explanted liver with Liver Imaging Reporting and Data System version 2017. Eur Radiol 2020;30:261-271

15. Cools KS, Moon AM, Burke LMB, McGinty KA, Strassle PD, Gerber DA. Validation of the liver imaging reporting and data system treatment response criteria after thermal ablation for hepatocellular carcinoma. Liver Transpl 2020;26:203-214

16. Kim SW, Joo I, Kim HC, Ahn SJ, Kang HJ, Jeon SK, et al. LI-RADS treatment response categorization on gadoxetic acid-enhanced MRI: diagnostic performance compared to mRECIST and added value of ancillary features. Eur Radiol 2020;30:2861-2870

17. Landis JR, Koch GG. The measurement of observer agreement for categorical data. Biometrics 1977;33:159-174

18. Kloeckner R, Otto G, Biesterfeld S, Oberholzer K, Dueber C, Pitton MB. MDCT versus MRI assessment of tumor response after transarterial chemoembolization for the treatment of hepatocellular carcinoma. Cardiovasc Intervent Radiol 2010;33:532-540

19. Amorim J, França M, Perez-Girbes A, Torregrosa A, Martí-Bonmatí L. Critical review of HCC imaging in the multidisciplinary setting: treatment allocation and evaluation of response. Abdom Radiol (NY) 2020;45:3119-3128

20. Semaan S, Makkar J, Lewis S, Chatterji M, Kim E, Taouli B. Imaging of hepatocellular carcinoma response after $90 \mathrm{Y}$ radioembolization. AJR Am J Roentgenol 2017;209:W263-W276

21. Min JH, Kang TW, Kim YY, Cha DI, Kim YK, Kim SH, et al. Vanishing washout of hepatocellular carcinoma according to the presence of hepatic steatosis: diagnostic performance of CT and MRI. Eur Radiol 2020 Nov [Epub]. https://doi. org/10.1007/s00330-020-07438-9

22. Kim DH, Choi SH, Byun JH, Kang JH, Lim YS, Lee SJ, et al. Arterial subtraction images of gadoxetate-enhanced MRI improve diagnosis of early-stage hepatocellular carcinoma. J Hepatol 2019;71:534-542 